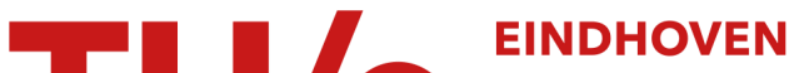

\section{Alignment probing of Rydberg states by stimulated emission}

\section{Citation for published version (APA):}

Spain, E. M., Dalberth, M. J., Kleiber, P. D., Leone, S. R., Op De Beek, S. S., \& Driessen, J. P. J. (1996).

Alignment probing of Rydberg states by stimulated emission. Journal of Chemical Physics, 102(24), 9522-9531.

https://doi.org/10.1063/1.468767

DOI:

10.1063/1.468767

Document status and date:

Published: 01/01/1996

\section{Document Version:}

Publisher's PDF, also known as Version of Record (includes final page, issue and volume numbers)

\section{Please check the document version of this publication:}

- A submitted manuscript is the version of the article upon submission and before peer-review. There can be important differences between the submitted version and the official published version of record. People interested in the research are advised to contact the author for the final version of the publication, or visit the $\mathrm{DOI}$ to the publisher's website.

- The final author version and the galley proof are versions of the publication after peer review.

- The final published version features the final layout of the paper including the volume, issue and page numbers.

Link to publication

\section{General rights}

Copyright and moral rights for the publications made accessible in the public portal are retained by the authors and/or other copyright owners and it is a condition of accessing publications that users recognise and abide by the legal requirements associated with these rights.

- Users may download and print one copy of any publication from the public portal for the purpose of private study or research.

- You may not further distribute the material or use it for any profit-making activity or commercial gain

- You may freely distribute the URL identifying the publication in the public portal.

If the publication is distributed under the terms of Article 25fa of the Dutch Copyright Act, indicated by the "Taverne" license above, please follow below link for the End User Agreement:

www.tue.nl/taverne

Take down policy

If you believe that this document breaches copyright please contact us at:

openaccess@tue.nl

providing details and we will investigate your claim. 


\title{
Alignment probing of Rydberg states by stimulated emission
}

\author{
Eileen M. Spain, ${ }^{\text {a) }}$ Mark J. Dalberth, Paul D. Kleiber, ${ }^{\text {b) }}$ and Stephen R. Leone ${ }^{\mathrm{c})}$ \\ Joint Institute for Laboratory Astrophysics, National Institute of Standards and Technology, \\ University of Colorado, Boulder, Colorado 80309 and Department of Chemistry and Biochemistry, \\ University of Colorado, Boulder, Colorado 80309
}

Stefan S. Op de Beek and Jan P. J. Driessen

Physics Department, Eindhoven University of Technology, P.O. Box 513, 5600 MB Eindhoven, The Netherlands

(Received 10 January 1995; accepted 22 March 1995)

\begin{abstract}
The possibility of probing the collisions of aligned Rydberg atoms by stimulated emission is assessed with studies of a polarized state and a new measurement of a collisional alignment effect in atomic $\mathrm{Ca}$. The stimulated emission method uses a laser to dump the desired state to a lower level which subsequently fluoresces. The technique can be used to obtain populations and polarization dependent information. First, the method is tested by applying it to an aligned $\mathrm{Ca}\left(4 s 17 d^{1} D_{2}\right)$ state. Alignment curves are measured when the initial state is prepared with both parallel and perpendicular relative polarizations. The experimentally observed alignment compares well with that derived from theoretical considerations of a saturated stimulated transition. Second, a two-vector collisional alignment experiment (initial state and relative velocity vector) is performed to study the energy transfer process $\mathrm{Ca}\left(4 s 7 d^{1} D_{2}\right)+\mathrm{He} \rightarrow \mathrm{Ca}\left(4 s 6 f{ }^{1} F_{3}\right)+\mathrm{He}+\Delta E=17.7 \mathrm{~cm}^{-1}$, and alignment effects are measured by both stimulated emission and conventional direct fluorescence detection. A preference for the $|m|=1$ and 2 initial states is observed in the relative cross sections. Essentially identical data are obtained with the two detection methods when elliptically polarized light is used for the stimulated emission detection method. The stimulated emission technique can provide alignment and population information of the final states, making it an excellent new tool for both three-vector correlation experiments and state-to-state Rydberg transitions. (C) 1995 American Institute of Physics.
\end{abstract}

\section{INTRODUCTION}

There is a vast body of experimental and theoretical work on the collisions of Rydberg atoms in the literature. ${ }^{1}$ The majority of these studies have focused on probing the scalar properties of these collisions. For example, the dependence of the cross sections for orbital angular momentum $(l)$ mixing, ${ }^{2,3}$ collisional depopulation (quenching), ${ }^{4-6}$ and collisional ionization ${ }^{6}$ on the principal quantum number $n$ of a Rydberg atom have been investigated. In addition, only a few studies have looked at state-to-state dynamics or alignment properties of Rydberg collisions. ${ }^{6-9}$ All of these studies have been important in understanding the collisional behavior of Rydberg atoms. Yet with modern laser probe methods a deeper understanding of Rydberg collisions is possible by addressing both the scalar and vector properties.

Consider the crossed beam atom-atom collision

$$
A^{*}(n, l)+\operatorname{Rg} \rightarrow A^{*}\left(n^{\prime}, l^{\prime}\right)+\operatorname{Rg}+\Delta E,
$$

where $\mathrm{Rg}$ is a rare gas and $A^{*}(n, l)$ is an excited and aligned Rydberg atom. If the initial Rydberg state is orbitally aligned by excitation with linearly polarized laser light, the dynamical event is intrinsically directional. To understand what effect this directionality has on the outcome of the collision, a vector correlation experiment is necessary. Such vector quan-

\footnotetext{
a) NSF Postdoctoral Fellow, Chemistry 1992-94.

${ }^{b}$ Permanent address: Department of Physics, University of Iowa, Iowa City, Iowa 52242.

${ }^{c)}$ Staff Member, Quantum Physics Division, NIST.
}

tities include initial and final relative velocities and initial and final state alignment. Understanding how the collision depends on the defined vector quantities allows the anisotropy of the collisional process to be unraveled. Such insight has been gleaned from energy transfer processes of initially aligned valence orbitals. Two-, three-, and four-vector correlations of aligned atomic valence states have been examined experimentally ${ }^{10-17}$ and theoretically. ${ }^{18-26}$ Recently, we are extending the studies of alignment effects to include statechanging collisions of Rydberg atoms. ${ }^{27}$

Quite different dynamics are possible for aligned Rydberg atoms compared to valence states because of their large spatial extent. Theoretical considerations of the collisional scattering of a Rydberg atom and a rare-gas atom have received intense scrutiny. The scattering event may be viewed as a three-body problem. Interactions occur among the Rydberg electron, the structureless rare-gas atom, and the ionic atomic core. The models describing Rydberg collisions rely on several approximations. One idea uses the BornOppenheimer approximation (BO) within certain limits when the electron moves much faster than the nuclei. ${ }^{9}$ However, since the electron velocity scales as $n^{-1}$, this approximation loses its validity for high Rydberg states. An alternative approach is based on a low electron velocity for large $n$ principal quantum numbers, in which the impulse approximation (IA) plus free electron model (i.e., low energy electron scattering) is appropriate. ${ }^{28-32}$ Morrison and co-workers applied this free electron model to the $\mathrm{Ca}+\mathrm{He}$ system and have pre- 


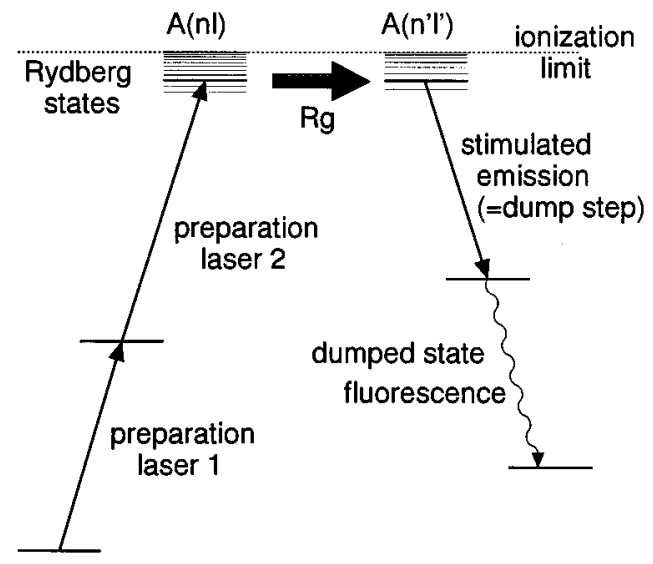

FIG. 1. Schematic representation of the energy levels for a Rydberg collision, where the final state is probed by the stimulated emission (termed the dump method in this paper). The subsequent dumped state fluorescence is measured in this experiment.

dicted about $10 \%$ alignment effects in a state-to-state energy transfer. $^{33}$

It might seem that to extend the study of orbital alignment effects to energy transfer processes of Rydberg states, the only requirement would be to excite to a higher energy state. However, because of the density of states in the Rydberg regime, detection of direct fluorescence from selected collisionally produced states is a nontrivial task. In addition, multiple collisions occur because of the long lifetime $\tau$ of the Rydberg states, and there is cascading through many levels. Here, a novel stimulated emission probing method to detect the final state is exploited, which is referred to as the dump method. Ongoing experiments in our laboratory have used this method to study a state-to-state collision-induced energy transfer of the Ca $4 s 17 d$ Rydberg state. ${ }^{27}$

In Fig. 1, a schematic energy diagram of a Rydberg collision is given, together with optical transitions used for both the preparation of the initial level and the stimulated emission dumping of the final state. The subsequent fluorescence from the dumped level is then measured and analyzed to obtain the collision cross sections as a function of initial orbital alignment. An advantage of the method is that all of the excitation and probe lasers are in the optical regime. States can be probed that are very near to the ionization limit, which would ordinarily require microwave frequencies for detection. Using nanosecond pulsed lasers, excellent time resolution is achieved, which can be used to avoid multiple collision events. Basically, the stimulated emission technique allows the resolution of many highly congested Rydberg levels. In addition, two-vector correlation experiments are readily extended to three-vector correlation studies, since the polarization vector of the dump laser can investigate the alignment (orientation) of the final state. In this paper we do not consider this supplemental feature of the final state alignment in the collision process.

Of course, the stimulated emission laser can ionize the $\mathrm{Ca}$ final state. Both photoionization and stimulated emission processes follow the rate expression for one-photon absorption

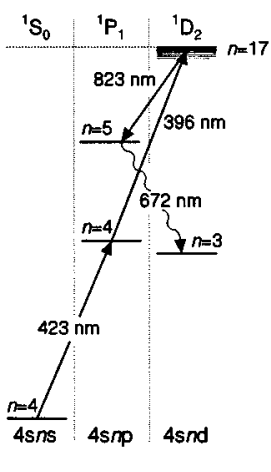

a) testing dump method

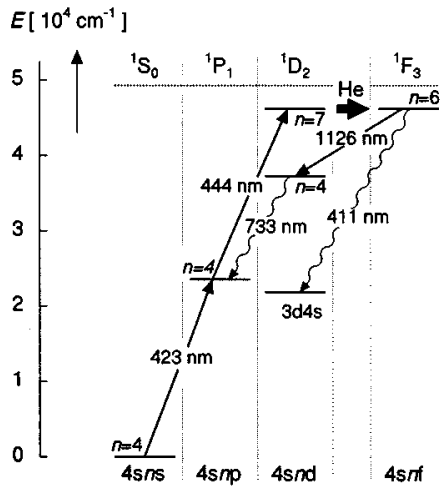

b) dump method vs. direct fluorescence

FIG. 2. Experiments to test the dump method. First, an initial aligned Rydberg state is (a) dumped. Second, a state-changing collision of $\mathrm{Ca}$ is investigated with (b) both the dump method and direct fluorescence. This allows for the dump method to be compared directly with the direct fluorescence technique, which no longer is applicable for Rydberg states.

$$
W_{f \leftarrow i}=\rho(\omega) B_{f i},
$$

where $\rho(\omega)$ is the energy density and $B_{f i}$ is the Einstein $B$ coefficient. Therefore, to assess the competition between photoionization and stimulated emission we need only compare the $B_{f i}$ values. The Einstein $B$ coefficients are

$$
B_{f i}=\frac{4 \pi^{2}}{3 \hbar^{2}}\left|\left\langle\psi_{f}|\hat{\mu}| \psi_{i}\right\rangle\right|^{2},
$$

where $\hat{\mu}$ is the transition dipole operator. Since the final state of the photoionization step is a continuum, whereas for the dump transition it is a bound state, the photoionization is much less likely than stimulated emission; this is evidenced in the experiment. We estimate from calcium cation signals that about 100 ions per laser pulse are produced by ionization of a $\mathrm{Ca} 4$ snd state. In fluorescence, however, we estimate that about $10^{5}$ or $10^{6}$ atoms are excited by the laser pulse and emit radiation.

The objective of this paper is to demonstrate and verify the use of an optical stimulated emission scheme as a probe for the Rydberg state population and alignment. In order to test this hypothesis, we perform two experiments which are shown in Fig. 2. First, we dump an initially aligned and excited $\left(4 s 17 d{ }^{1} D_{2}\right)$ level of $\mathrm{Ca}$ to the $\left(4 s 5 p{ }^{1} P_{1}\right)$ state using an $823 \mathrm{~nm}$ laser photon [Fig. 2(a)]. The subsequent dumped state fluorescence to the $\left(3 d 4 s^{1} D_{2}\right)$ state at 672 $\mathrm{nm}$ is then collected in a well-defined direction. Notice that no collisional scattering occurs in this polarization probe example. The dumped state fluorescence is measured as a function of the arrangement of the three linear laser polarizations with respect to the viewing direction. We compare these results to theoretical predictions of the alignment effect. Because the dump method may introduce extra alignment (or orientation) in the dumped state, the effects of anisotropy in the emitted dumped state fluorescence and saturation of the dump transition are taken into account in the analysis.

Second, we use the dump method to measure an alignment effect of an unexplored energy transfer process; as a check, we interrogate the system both by stimulated emission 


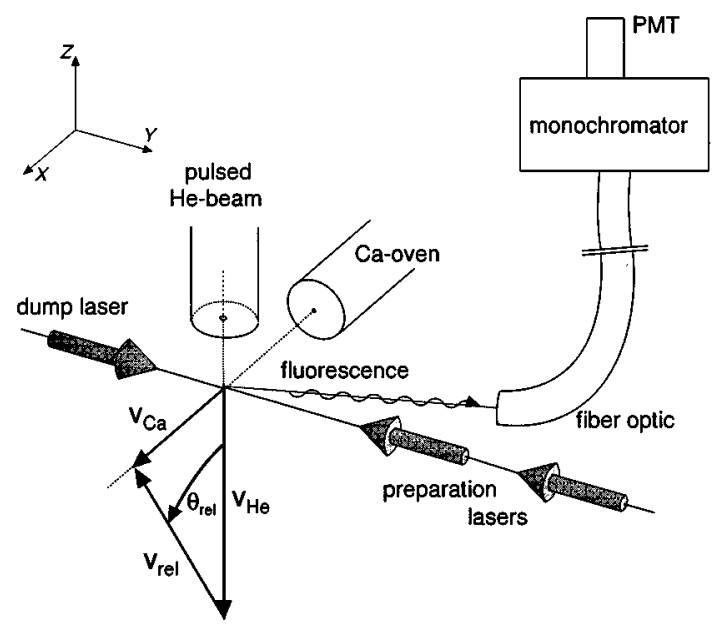

FIG. 3. Schematic view of the experimental setup used to test the dump method. A space-fixed coordinate frame $(X, Y, Z)$ is introduced, with the $X$ axis coinciding with the $\mathrm{Ca}$ beam, the $Z$ axis corresponding with the $\mathrm{He}$ beam. The laser beams used for preparing and dumping the Ca states are counterpropagating along the $Y$ axis. The fiber bundle collects the dumped state fluorescence, and light is routed to a monochromator and a photomultiplier tube (PMT)

and the more conventional direct fluorescence detection methods. An initial $\mathrm{Ca}\left(4 s 7 d{ }^{1} D_{2}\right)$ state is excited, which can be probed by both methods. Collision-induced transfer into the $\left(4 s 6 f{ }^{1} F_{3}\right)$ state by $\mathrm{He}$ is studied with the two different schemes. In one experiment, the final state $\left({ }^{1} F_{3}\right)$ is dumped down to the $\left(4 s 4 d{ }^{1} D_{2}\right)$ state using an $1126 \mathrm{~nm}$ photon and the subsequent emission to the $\left(4 s 4 p{ }^{1} P_{1}\right)$ at $733 \mathrm{~nm}$ is measured as shown in Fig. 2(b). Elliptically polarized laser light is used in this dump step to assure that all magnetic sublevels of the final $(4 s 6 f)$ state are dumped equally in saturation. Because the final state alignment (orientation) can be ignored in this case, a two-vector correlation experiment is achieved (initial alignment vs relative velocity), and the results of the dump method can be compared directly with those from conventional direct fluorescence detection. Here, spontaneous emission of the final $\left({ }^{1} F_{3}\right)$ level to the $\left(3 d 4 s{ }^{1} D_{2}\right)$ state at $411 \mathrm{~nm}$ is monitored, as depicted in Fig. 2(b). Interpretation of the data from the collisioninduced energy transfer will be presented.

\section{EXPERIMENTAL METHOD}

\section{A. Overview}

The experimental setup is shown schematically in Fig. 3 and consists of an effusive beam of $\mathrm{Ca}$, a pulsed supersonic jet of $\mathrm{He}$, two collinear tunable pulsed laser beams to prepare the initial state, and a third tunable pulsed dye laser, collinear and counterpropagating with the excitation laser beams, to stimulate emission from any state. The $\mathrm{Ca}$ and $\mathrm{He}$ beams are crossed perpendicularly to form a well defined initial relative velocity vector or collision axis, $v_{\text {rel }}$. Fluorescence is detected through a fiber optic bundle, at an angle of $68^{\circ}$ from the Ca beam axis (see Fig. 3), and monochromator plus photomultiplier tube (PMT) or a fiber optic bundle and interference filter plus PMT arrangement. The fluorescence signal

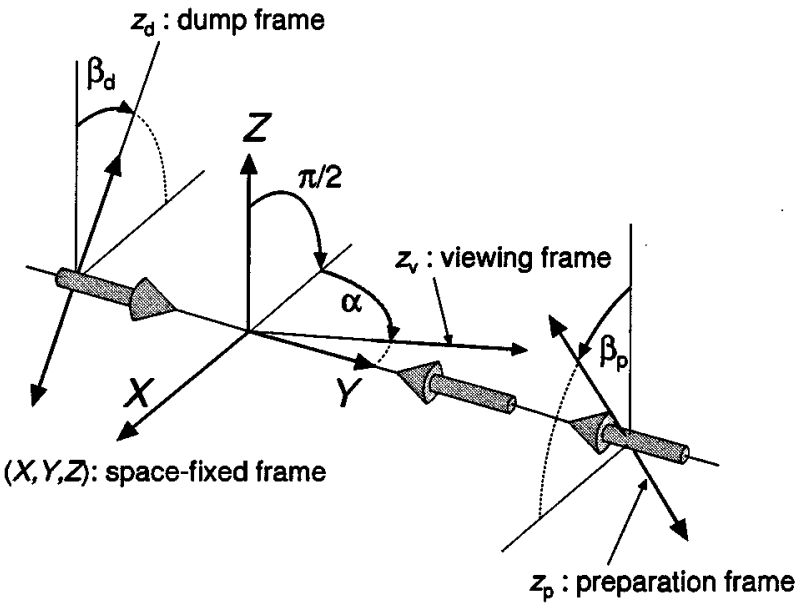

FIG. 4. Angular configuration of the vector quantities, relevant for testing the dump method. The Ca Rydberg state is described initially in the preparation frame (angle $\beta_{p}$ ). The stimulated emission process is represented in the dump frame (angle $\beta_{d}$ ), and finally, a viewing frame (angles $\pi / 2, \alpha$ ) is introduced to describe the collected dumped state fluorescence.

from stimulated emission or direct fluorescence is detected as a function of the angle between the polarization of the excitation lasers and $v_{\text {rel }}$.

\section{B. Stimulated emission detection of aligned Ca $4 s 17 d$}

In order to investigate the possibility of measuring reliable final state populations with the dump method, we stimulate emission from an initially aligned and excited $\mathrm{Ca}\left(4 s 17 d{ }^{1} D_{2}\right)$ level to the $\left(4 s 5 p{ }^{1} P_{1}\right)$ state using an 823 $\mathrm{nm}$ laser photon [Fig. 2(a)]. The subsequent dumped state fluorescence to the $\left(3 d 4 s^{1} D_{2}\right)$ state at $672 \mathrm{~nm}$ is then collected in a well-defined direction and compared to theory as a function of the initial state polarization. The three relevant vector quantities in this experimental test of the dump method are shown in Figs. 3 and 4. There are two polarization vectors of the laser beams (423 and $396 \mathrm{~nm}$ ) which prepare the initial state and one polarization vector of the laser beam $(823 \mathrm{~nm})$ which stimulates emission from the aligned Ca state. Typically, an aligned (oriented) lower state is populated in the dump process. Finally, the subsequent dumped state fluorescence serves as an indicator for the upper state population, which is detected in a well-defined direction determined by the position of the fiber bundle that collects the photons. This viewing direction determines the third relevant vector quantity. Note that the velocity vectors of the Ca beam (and He beam) are not relevant here, since no collisional scattering occurs in this test of the stimulated emission technique.

The general angular configuration of the relevant vectors is also depicted in Fig. 4. A space-fixed coordinate frame $(X, Y, Z)$ is used as a reference frame, which is also schematically shown in Fig. 3. The collinear excitation lasers are linearly polarized and the polarizations are arranged either parallel or perpendicular to each another. Both polarizations can be simultaneously rotated in the $X-Z$ plane. The initial $\mathrm{Ca}\left(4 s 17 d{ }^{1} D_{2}\right)$ state is described most easily in a coordi- 
nate frame where one of the laser polarization vectors serves as the quantization axis. We term this coordinate frame the preparation frame $\left(x_{p}, y_{p}, z_{p}\right)$. The laser fixed $z_{p}$ axis makes an angle $\beta_{p}$ with respect to the space-fixed $Z$ axis as visualized in Fig. 4. The linearly polarized dump laser is collinear and counterpropagating with the two excitation laser beams. Therefore, the polarization vector of the dump laser is rotated in the same $X-Z$ plane, making an angle $\beta_{d}$ with the $Z$ axis as shown in Fig. 4. This laser polarization serves as the quantization axis in the dump frame $\left(x_{d}, y_{d}, z_{d}\right)$. The fiber optic bundle that collects the dumped state fluorescence is positioned in the space-fixed $X-Y$ plane, making an angle $\alpha=68^{\circ}$ with respect to the negative $X$ axis. This viewing direction defines a corresponding viewing frame $\left(x_{v}, y_{v}, z_{v}\right)$ with the $z_{v}$ axis coinciding with the viewing direction, as is depicted in Fig. 4.

The $4 s 17 d$ is prepared by a two-step absorption of linearly polarized light produced by the $355 \mathrm{~nm}$ output of a single Nd:YAG laser pumping two tandem pulsed dye lasers [423 and $396 \mathrm{~nm}$ as noted in Fig. 2(a)]. Two distinct arrangements of the linear polarizations of the combined excitation laser beams are chosen. First, the two laser beams are combined and passed through a single Glan prism. In such an arrangement, the two laser polarizations are linear and parallel, so termed the parallel polarization $(\uparrow \uparrow)$. Second, one laser beam is passed through a half-wave plate to rotate the beam $90^{\circ}$ prior to beam combination. Then the two laser polarizations are linear and perpendicular $(\uparrow \leftrightarrow)$. The two beams are combined and sent through a double Fresnel rhomb polarization rotator, which permits the angle, $\beta_{p}$, between the pump polarization and the space-fixed $Z$ axis to be varied.

The orbitally aligned $\left(4 s 17 d^{1} D_{2}\right)$ state is deexcited to the $\left(4 s 5 p{ }^{1} P_{1}\right)$ state using an $823 \mathrm{~nm}$ dump photon produced by a dye laser pumped by the second harmonic output of the second Nd:YAG. The dump laser polarization is linear and fixed perpendicular to the atomic $\mathrm{Ca}$ beam axis. This means that the dump frame $\left(x_{d}, y_{d}, z_{d}\right)$ coincides with the space-fixed frame $(X, Y, Z)$, and we can set $\beta_{p}=\beta$ and $\beta_{d}=0$ in our analysis. The pump-probe delay was set at $100 \mathrm{~ns}$. The subsequent dumped state fluorescence to the $\left(3 d 4 s{ }^{1} D_{2}\right)$ state at $672 \mathrm{~nm}$ is then measured with a fiber optic bundle coupled to a monochromator and photomultiplier tube (PMT). The $672 \mathrm{~nm}$ signal is averaged with a boxcar.

\section{C. $4 s 7 d$ collision-induced energy transfer}

A collisional experiment is also performed in the crossed-beam apparatus shown in Fig. 3, where the initial relative velocity vector, $v_{\text {rel }}$, is defined by the crossed atomic beams. The collision energy is $67 \mathrm{meV}$ for the $\mathrm{Ca}+\mathrm{He}$ system. The relative polarizations of the lasers are prepared with half-wave plates and Glan prisms; the combined excitation laser beams are propagated through a double Fresnel rhomb polarization rotator, as described in Sec. II B. Here, however, the angle $\beta_{\text {rel }}$ between the initial state alignment and the relative velocity vector is varied in the alignment experiment (see Figs. 3 and $4, \beta_{\text {rel }}=\beta_{p}+\theta_{\text {rel }}$ ).
Two distinct experiments are performed using two different detection methods. In the first experiment, a third laser beam stimulates emission down from the $\left(4 s 6 f{ }^{1} F_{3}\right)$ state to a $\left(4 s 4 d^{1} D_{2}\right)$ state and the subsequent emission to the $\left(4 s 4 p{ }^{1} P_{1}\right)$ state at $733 \mathrm{~nm}$ is measured as shown in Fig. 2(b). The second experiment uses fluorescence detection; the $411 \mathrm{~nm}$ spontaneous emission from the final $\left(4 s 6 f^{1} F_{3}\right)$ state to the $\left(3 d 4 s^{1} D_{2}\right)$ state is monitored as displayed in Fig. 2(b). For each experiment, the initial $\left(4 s 7 d^{1} D_{2}\right)$ state is prepared with two dye laser pulses, one for excitation to the $\left(4 s 4 p{ }^{1} P_{1}\right)$ state at $423 \mathrm{~nm}$ and one for excitation to the $\left(4 s 7 d{ }^{1} D_{2}\right)$ state at $444 \mathrm{~nm}$. These two collinear pulsed dye laser beams are propagated perpendicular to the plane containing the two atomic beams. The dye lasers are arranged in tandem and are pumped by the $355 \mathrm{~nm}$ output of a single Nd:YAG laser. The two laser beams are combined before entrance to the chamber by a dichroic beam splitter. The dump laser beam is produced from the output of a third dye laser pumped by the $532 \mathrm{~nm}$ output of a second $\mathrm{Nd}$ :YAG laser.

\section{Stimulated emission detection}

To stimulate emission from the final $\left(4 s 6 f^{1} F_{3}\right)$ state to the $\left(4 s 4 d{ }^{1} D_{2}\right)$ state, a wavelength of $1125.6 \mathrm{~nm}$ is required. The $532 \mathrm{~nm}$ output of a second Nd:YAG laser pumps a third pulsed dye laser. The output of the dye laser is directed through a Raman cell containing $\mathrm{H}_{2}$ at a pressure of $4 \times 10^{6} \mathrm{~Pa}$. The Raman first stokes radiation is produced at $1125.6 \mathrm{~nm}$ and separated from the fundamental and antiStokes radiation by a Pellin-Broca prism. The $1125.6 \mathrm{~nm}$ radiation (approximately $200 \mu \mathrm{J} / \mathrm{pulse}$ ) is directed into the vacuum chamber collinear and counterpropagating to the excitation laser beams. The stimulated emission laser pulse is delayed $100 \mathrm{~ns}$ from the pump laser trigger. The radiation is linearly polarized with respect to the space-fixed $Z$ axis. To produce an elliptically polarized dump pulse, the linearly polarized radiation is propagated through a Babinet-Soliel Compensator set to act as a $\lambda / 8$ wave plate. Fluorescence from the $\left(4 s 4 d{ }^{1} D_{2}\right)$ to the $\left(4 s 4 p{ }^{1} P_{1}\right)$ state at $733 \mathrm{~nm}$ is measured at a gate acceptance delay of $175 \mathrm{~ns}$ after the pump laser trigger, with a width of $75 \mathrm{~ns}$. One fiber optic bundle couples light to the monochromator plus PMT, the signal is amplified, and $733 \mathrm{~nm}$ photons are counted. The initial state fluorescence, monitored for normalization purposes at 672 $\mathrm{nm}$ with an interference filter and PMT, is amplified for boxcar integration.

\section{Direct fluorescence detection}

The energy transfer process is detected by measuring the fluorescence from the $\left(4 s 6 f^{1} F_{3}\right)$ state at $411 \mathrm{~nm}$. The fluorescence signal is collected by a fiber optic bundle coupled to a $0.5 \mathrm{~m}$ monochromator and photomultiplier tube (PMT). The PMT current is amplified and integrated with a boxcar. Time-gated detection with boxcar integration is used for processing the final state fluorescence. The boxcar gate is delayed by $100 \mathrm{~ns}$ from the laser trigger and has an acceptance width of 100 ns. Background signals are accounted for by blocking the combined excitation laser beam and subtracting 
the resulting background counts. Signals are normalized for the initial state fluorescence by time-gated boxcar integration of emission at $672 \mathrm{~nm}$, arising by cascade from the initial $\left(4 s 7 d^{1} D_{2}\right)$ state to the $\left(4 s 5 p{ }^{1} P_{1}\right)$ state and subsequent emission down to the $\left(3 d 4 s^{1} D_{2}\right)$ state. A second fiber optic bundle collects direct fluorescence from the initial ${ }^{1} D_{2}$ state, selected by a bandpass filter at $672 \mathrm{~nm}$, and the signal is processed through an amplifier and boxcar.

\section{VERIFICATION OF THE STIMULATED EMISSION METHOD USING THE POLARIZED $\mathrm{Ca}\left(4 s 17 d^{1} D_{2}\right)$}

\section{A. Theory of dump detection}

\section{Upper state}

The pure initial state, $\mathrm{Ca}\left(4 s 17 d^{1} D_{2}\right)$, that serves as the upper state $\left|\psi_{u}\right\rangle$ for the dumping process, can be represented by a vector in the $\left(2 j_{u}+1\right)$ dimensional space with respect to the laser quantization axis $z_{p}$ :

$$
\left|\psi_{u}\right\rangle_{z_{p}}=\sum_{m} a_{m}\left|j_{u}, m\right\rangle_{z_{p}}=\left(\begin{array}{c}
a_{-j_{u}} \\
a_{-j_{u}+1} \\
\vdots \\
a_{j_{u}}
\end{array}\right),
$$

where the $a_{m}$ are the expansion coefficients. Two different initial states can be created when the two linear laser polarizations are (i) parallel $(\uparrow \uparrow)$ or (ii) perpendicular $(\uparrow \leftrightarrow)$, producing states denoted as $\left|\psi_{u, \|}\right\rangle$ and $\left|\psi_{u, \perp}\right\rangle$. In the preparation frame the two states have the following vector representations:

$$
\left|\psi_{u, \|}\right\rangle_{z_{p}}=\left(\begin{array}{c}
0 \\
0 \\
1 \\
0 \\
0
\end{array}\right), \quad\left|\psi_{u, \perp}\right\rangle_{z_{p}}=\frac{1}{\sqrt{2}}\left(\begin{array}{c}
0 \\
1 \\
0 \\
-1 \\
0
\end{array}\right) .
$$

In order to calculate the form of the dumped lower level $\left|\psi_{l}\right\rangle$, the upper state is represented in the dump frame, which coincides with the space-fixed frame in our experiment $\left(\beta_{p}=\beta\right.$, $\left.\beta_{d}=0\right)$. For this we use the rotation matrix elements $D_{m, m^{\prime}}^{j}(0, \beta, 0)$, given by the Wigner- $d$ functions $d_{m, m^{\prime}}^{j}(\beta)::^{34,35}$

$$
\begin{aligned}
& \left|\psi_{u, \|}\right\rangle_{z_{d}}=\sum_{m_{u}} d_{m_{u}, 0}^{j_{u}}(\beta)\left|j_{u} m_{u}\right\rangle_{z_{d}}, \\
& \left|\psi_{u, \perp}\right\rangle_{z_{d}}=\sum_{m_{u}} \frac{1}{\sqrt{2}}\left[d_{m_{u},-1}^{j_{u}}(\beta)-d_{m_{u}, 1}^{j_{u}}(\beta)\right]\left|j_{u} m_{u}\right\rangle_{z_{d}} .
\end{aligned}
$$

\section{Stimulated emission or dump step}

In the dump frame the upper level is given by the superposition states of Eq. (6). We derive a general expression for a lower state which is stimulated down from an upper superposition state with a linearly polarized photon. Two extreme situations are considered in this derivation, i.e., for low laser powers the transitions are unsaturated, whereas for high laser powers they are saturated. The laser powers that define the unsaturated and saturated limits depend on the particular optical transitions under consideration. Both situations are considered in the following analysis.

The time-dependent electromagnetic field $\mathbf{E}(t)=\mathbf{E} e^{-i \omega t}$ perturbs the Hamiltonian $\mathscr{H}^{(0)}$ describing the atomic system. In first order this results in the dipole operator,

$$
\hat{\mathscr{H}}^{(1)}(t)=-\mathbf{E}(t) \cdot \hat{\mu}=e\left(E_{x} \hat{x}+E_{y} \hat{y}+E_{z} \hat{z}\right) e^{-i \omega t},
$$

where $\hat{\mu}$ is the electric dipole moment operator and the righthand side is written in a Cartesian basis $(\hat{x}, \hat{y}, \hat{z})$. A more suitable basis set would be $(\hat{x}-i \hat{y}, \hat{z}, \hat{x}+i \hat{y})=\left(\hat{e}_{-1}^{1}, \hat{e}_{0}^{1}, \hat{e}_{1}^{1}\right)$, which transforms under rotations like spherical harmonics $Y_{1 m}$.

The transition matrix elements of an upper state $\left|j_{u} m_{u}\right\rangle$ into a lower state $\left|j_{l} m_{l}\right\rangle$ are calculated in the dump frame $\left(x_{d}, y_{d}, z_{d}\right)$, where we have a laser polarization $\hat{E}_{m}=E \hat{e}_{m}^{1}$ with $m=0$ and $m= \pm 1$ denoting linear and circular polarizations, respectively. This results in

$$
\left\langle j_{l} m_{l}\left|E \hat{e}_{m}^{1}\right| j_{u} m_{u}\right\rangle=\left\langle 1 m j_{l} m_{l} \mid j_{u} m_{u}\right\rangle\left\langle j_{l}\left\|E^{1}\right\| j_{u}\right\rangle,
$$

where we have applied the Wigner-Eckart theorem. ${ }^{34}$ In this equation $\left\langle j_{l}\left\|E^{1}\right\| j_{u}\right\rangle$ is the reduced matrix element, independent of the magnetic sublevel quantum numbers $m_{l}$ and $m_{u}$, and $\left\langle 1 m j_{l} m_{l} \mid j_{u} m_{u}\right\rangle$ is a geometrical Clebsch-Gordan coefficient. ${ }^{34,35}$

In our experiment the upper state is not a single magnetic sublevel with respect to the dump laser polarization, but a coherent superposition state as given by Eqs. (4), (5), and (6):

$$
\left|\psi_{u}\right\rangle_{z_{d}}=\sum_{m_{u}} a_{m_{u}}\left|j_{u} m_{u}\right\rangle_{z_{d}} .
$$

In order to calculate the dumped lower level we have to consider two approximations. First, we will take into account the stimulated emission process by a $|1, m\rangle$ photon only and neglect the reverse process, i.e., the absorption of a $|1, m\rangle$ photon by the lower level. In this so-called unsaturated situation the lower state $\left|\psi_{l}\right\rangle$, is given by

$$
\begin{aligned}
\left|\psi_{l}^{\mathrm{uns}}\right\rangle_{z_{d}} & =\sum_{m_{l}} \sum_{m_{u}} a_{m_{u}}\left|j_{l} m_{l}\right\rangle_{z_{d}}\left\langle j_{l} m_{l}\left|E \hat{e}_{m}^{1}\right| j_{u} m_{u}\right\rangle \\
& =\sum_{m_{l}} \sum_{m_{u}} a_{m_{u}}\left|j_{l} m_{l}\right\rangle_{z_{d}}\left\langle 1 m j_{l} m_{l} \mid j_{u} m_{u}\right\rangle\left\langle j_{l}\left\|E^{1}\right\| j_{u}\right\rangle .
\end{aligned}
$$

However, in the second approximation the lower level is populated significantly and the absorption of a dump laser photon by the lower state $\left|\psi_{l}\right\rangle$ has to be taken into account as well. In the saturated case, the magnetic sublevels which are connected in the optical transitions are equally populated and we no longer have to consider the geometrical ClebschGordan coefficients. In this saturated condition the lower level is given by

$$
\left|\psi_{l}^{\text {sat }}\right\rangle_{z_{d}}=\sum_{m_{l}} \frac{1}{2} a_{m_{l}}\left|j_{l} m_{l}\right\rangle_{z_{d}}
$$


where the sum over $m_{l}$ only runs over those magnetic sublevels for which the optical transition is allowed, i.e., for which the Clebsch-Gordan coefficient $\left\langle 1 m j_{l} m_{l} \mid j_{u} m_{u}\right\rangle$ is essentially nonzero.

The orbitally aligned $\left(4 s 17 d^{1} D_{2}\right)$ state $\left(j_{u}=2\right)$ is stimulated to the $\left(4 s 5 p{ }^{1} P_{1}\right)$ state $\left(j_{l}=1\right)$ using an $823 \mathrm{~nm}$ dump photon. In our experiment the linearly polarized dump photon is given by the vector $|1,0\rangle$ in the space-fixed frame. In the unsaturated case the lower states, $\left|\psi_{l, \|}^{\text {uns }}\right\rangle$ and $\left|\psi_{l, \perp}^{\text {uns }}\right\rangle$, are given by

$$
\begin{aligned}
\left|\psi_{l, \|}^{\mathrm{uns}}\right\rangle_{z_{d}}= & \sum_{m_{l}} d_{m_{l}, 0}^{j_{u}}(\beta)\left\langle 10 j_{l} m_{l} \mid j_{u} m_{l}\right\rangle\left|j_{l} m_{l}\right\rangle_{z_{d}}, \\
\left|\psi_{l, \perp}^{\mathrm{uns}}\right\rangle_{z_{d}}= & \sum_{m_{l}}(1 / \sqrt{2})\left[d_{m_{l},-1}^{j_{u}}(\beta)-d_{m_{l}, 1}^{j_{u}}(\beta)\right] \\
& \times\left\langle 10 j_{l} m_{l} \mid j_{u} m_{l}\right\rangle\left|j_{l} m_{l}\right\rangle_{z_{d}} .
\end{aligned}
$$

In the saturated situation, the magnetic sublevels which are connected in the optical transitions are populated equally and the geometrical Clebsch-Gordan coefficients no longer appear in the expression of the lower state. In this saturated condition the lower levels $\left|\psi_{l, \|}^{\text {sat }}\right\rangle$ and $\left|\psi_{l, \perp}^{\text {sat }}\right\rangle$ are given by

$$
\begin{aligned}
\left|\psi_{l, \|}^{\mathrm{sat}}\right\rangle_{z_{d}} & =\sum_{m_{l}} d_{m_{l}, 0}^{j_{u}}(\beta)\left|j_{l} m_{l}\right\rangle_{z_{d}}, \\
\left|\psi_{l, \perp}^{\mathrm{sat}}\right\rangle_{z_{d}} & =\sum_{m_{l}}(1 / \sqrt{2})\left[d_{m_{l},-1}^{j_{u}}(\beta)-d_{m_{l}, 1}^{j_{u}}(\beta)\right]\left|j_{l} m_{l}\right\rangle_{z_{d}},
\end{aligned}
$$

where the summation runs over all magnetic sublevels $m_{l}$ for which the optical transitions, $\left|j_{u} m_{u}\right\rangle \rightarrow\left|j_{l} m_{l}\right\rangle$, are allowed by selection rules.

\section{Dumped state fluorescence}

The dumped state fluorescence from the $\left(4 s 5 p{ }^{1} P_{1}\right)$ state to the $\left(3 d 4 s^{1} D_{2}\right)$ state at $672 \mathrm{~nm}$ is monitored with a fiber optic bundle coupled to a monochromator and a PMT. The photons emitted in the $\hat{z}_{v}$ direction of the viewing frame are detected. This direction corresponds to the $68^{\circ}$ angle that the fiber optic bundle makes with the $\mathrm{Ca}$ beam axis. Only the two circular laser polarizations have to be considered in this frame, i.e., $\hat{e}_{-1}^{1}$ and $\hat{e}_{1}^{1}$. Therefore, we transform the lower states $\left|\psi_{l}\right\rangle$ of Eqs. (12) and (13) into the viewing frame. Since the dump frame coincides with the space-fixed frame, only a rotation over the Euler angles $(\alpha, \pi / 2,0)$ is necessary. The net result of this rotation is

$$
\left|j_{l} m_{l}\right\rangle_{z_{d}}=\sum_{m_{l}^{\prime}} D_{m_{l}^{\prime}, m_{l}}^{j_{l}}\left(\alpha, \frac{\pi}{2}, 0\right)\left|1 m_{l}^{\prime}\right\rangle_{z_{v}}
$$

Combining Eqs. (12), (13), and (14) allows us to represent the deexcited $\mathrm{Ca}\left(4 s 5 p^{1} P_{1}\right)$ state in the viewing frame for both the parallel and the perpendicular orbital preparation and for both the saturated and unsaturated dump transition. The solid angle of collection of the fiber optic bundle is not included in the calculation. It has been shown ${ }^{36}$ that the fluorescence anisotropy for a perfectly aligned atomic state is not critically dependent on the finite solid angle of collection.

To calculate the spontaneous emission rate for the two possible photon polarizations, $|1,-1\rangle$ and $|1,+1\rangle$, we make use of the transition matrix elements of Eq. (8) connecting the $\left(4 s 5 p{ }^{1} P_{1}\right)$ state $\left(j_{l}=1\right)$ to the $\left(3 d 4 s{ }^{1} D_{2}\right)$ state $\left(j_{f}=2\right)$

$$
\begin{aligned}
I_{\mathrm{ph}}= & \sum_{m_{l}=-1}^{+1}\left[\left|\left\langle 1-1 j_{f}\left(m_{l}+1\right) \mid j_{l} m_{l}\right\rangle\right|^{2}+\mid\langle 1+1\right. \\
& \left.\times\left. j_{f}\left(m_{l}-1\right)\left|j_{l} m_{l}\right\rangle\right|^{2}\right]\left|\left\langle j_{f}\left\|E^{1}\right\| j_{l}\right\rangle\right|^{2}\left|\left\langle j_{l} m_{l} \mid \psi_{l}\right\rangle\right|^{2},
\end{aligned}
$$

where $l$ labels the lower level of the dump step from which the dumped state fluorescence originates and $f$ labels the final level of the dumped state transition. The dumped state $\left|\psi_{l}\right\rangle$ can be represented in the viewing frame by combining Eqs. (12), (13), and (14), resulting in analytical expressions for the bracket $\left\langle j_{l} m_{l} \mid \psi_{l}\right\rangle$.

In this verification of the stimulated emission technique we prepare two different initial wave functions with parallel $(\uparrow \uparrow)$ or perpendicular $(\uparrow \leftrightarrow)$ laser polarizations. Furthermore, the dump transition can be either saturated (sat) for high laser powers, or unsaturated (uns) for low laser powers. Combination of Eqs. (12), (13), (14), and (15) results in the four following (normalized) expressions:

$$
\begin{aligned}
& \|, \text { uns } I_{\mathrm{ph}}=\frac{542-18 \cos 2 \alpha(1-\cos 4 \beta)+336 \cos 2 \beta+18 \cos 4 \beta}{542-18 \cos 2 \alpha} \\
& \| \text {, sat } I_{\mathrm{ph}}=\frac{155-6 \cos 2 \alpha(1-\cos 4 \beta)+84 \cos 2 \beta-15 \cos 4 \beta}{155-6 \cos 2 \alpha}, \\
& \perp, \text { uns } I_{\mathrm{ph}}=\frac{56-2(1+\cos 2 \alpha)(1+\cos 4 \beta)}{54-2 \cos 2 \alpha} \\
& \perp \text {, sat } I_{\mathrm{ph}}=\frac{47-2 \cos 2 \alpha(1+\cos 4 \beta)+5 \cos 4 \beta}{47-2 \cos 2 \alpha} .
\end{aligned}
$$



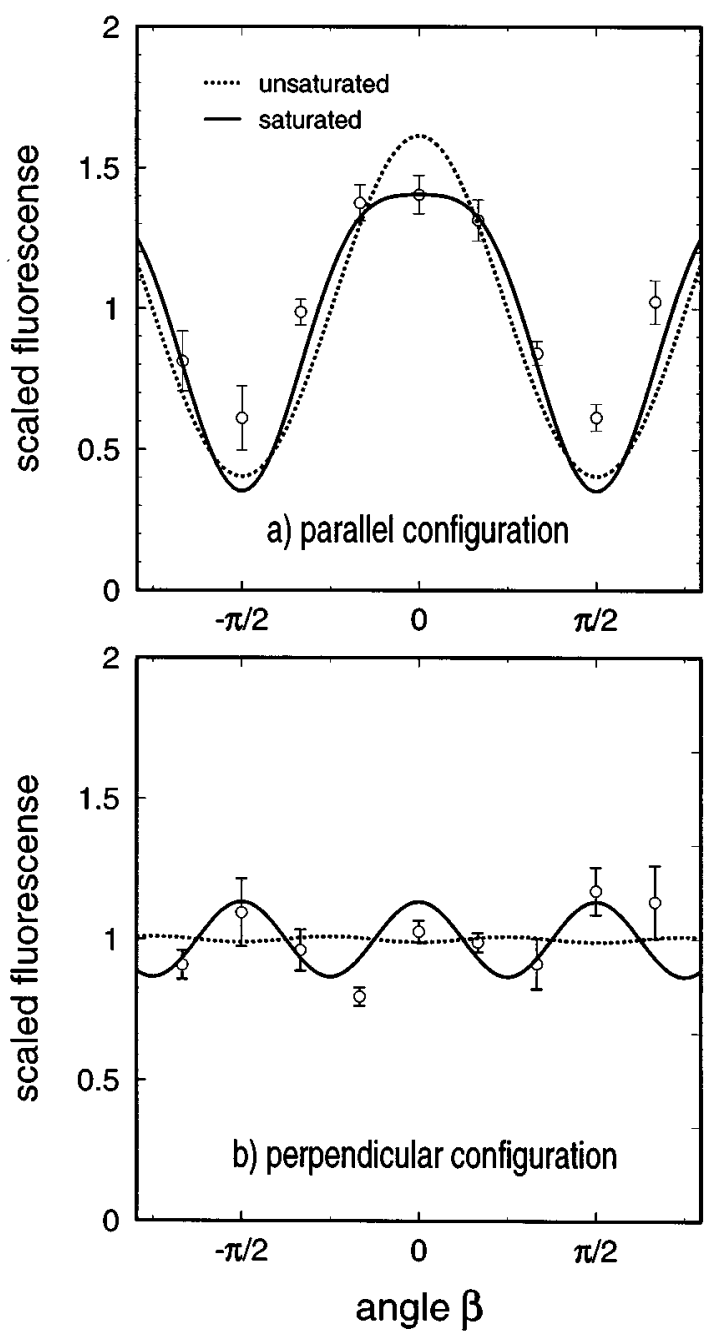

FIG. 5. Alignment data for dumping the aligned $\mathrm{Ca}\left(4 s 17 d{ }^{1} D_{2}\right)$ state, resulting from multiple coadditions. The two preparation lasers are aligned (a) parallel and (b) perpendicular. The normalized theoretical curves of Eq. (16) are shown as well. In both configurations the saturated model gives better agreement than the unsaturated case.

Putting in the experimental value, $\alpha=68^{\circ}$, gives the final equations which can be used in a least-squares fit of the data, which we obtain by rotating the laser polarizations of the preparation lasers about the $\mathrm{Ca}$ beam axis.

\section{B. Results and discussion of Ca $4 s 17 d{ }^{1} D_{2}$ stimulated emission probing}

In Fig. 5, we compare the experimental alignment curve for dumping an aligned $\left(4 s 17 d^{1} D_{2}\right)$ state to the theoretical predictions generated by Eq. (16) for the saturated and unsaturated cases. In these measurements the linear dump laser polarization has been fixed parallel to the space-fixed $Z$ axis, $\beta_{d}=0$, and only the parallel (or perpendicular) polarizations of the two preparation lasers are rotated in the $X-Z$ plane, $\beta_{p}=\beta$. Alignment curves are taken over a range of nine angles for $\beta$ in steps of $30^{\circ}$ from $-120^{\circ}$ to $+120^{\circ}$. To ensure that fluctuations of the $\mathrm{Ca}$ beam from different daily runs and the drift of the time gating do not degrade the angular structure, each alignment curve is normalized to its own average. For parallel laser polarizations eleven alignment curves are averaged to yield the final result, whereas for the perpendicular polarizations only four alignment curves are combined.

Figure 5(a) displays the observed signals obtained for the parallel case, together with the theoretical curves obtained with the expressions of Eq. (16), for the saturated and unsaturated cases. Both expressions predict a large alignment effect, in agreement with the observed alignment data. However, the saturated case shows better overall agreement. This is not a surprise, since the dump laser power was maximized in order to obtain the largest signal counts for the dumped state fluorescence. In Fig. 5(b) the experimental data for the perpendicular case are shown, together with the theoretical predictions. The theoretical expression predicts a much smaller alignment effect, resulting in a very different vertical scale. Notice, however, that the error bars, which are obtained by averaging four data sets, are of the same order of magnitude as in the parallel case. Again, better agreement is observed for the saturated case. In the saturated cases, the slight disagreement between the experimental and theoretical alignment effects may be attributed to the exclusion of the finite solid angle of collection by the fiber optic in the theory. We estimate a 5\%-10\% difference between the experimental and theoretical curves by ignoring the finite solid angle.

\section{COLLISIONAL ALIGNMENT EFFECT OF $\mathrm{Ca}\left(4 s 7 d^{1} D_{2}\right)+$ He ENERGY TRANSFER}

\section{A. Dump method vs direct fluorescence}

In this section we use the stimulated emission technique to measure a collision-induced energy transfer alignment effect. The ultimate goal is to investigate alignment effects for the collisional energy transfer of Rydberg states. To provide a first measurement that can be compared to the conventional fluorescence methods used previously, we choose a system where the initial state is neither strictly in the valence nor in the Rydberg regime, but which can be probed by both methods. The system is

$$
\begin{aligned}
& \mathrm{Ca}\left(4 s 7 d{ }^{1} D_{2}\right)+\mathrm{He} \rightarrow \mathrm{Ca}\left(4 s 6 f^{1} F_{3}\right)+\mathrm{He}+\Delta E \\
& \quad=17.7 \mathrm{~cm}^{-1} .
\end{aligned}
$$

\section{Two- and three-vector correlations}

Although not obtained here, in principle, the stimulated emission technique could provide alignment information of the final state. The polarized dump laser would be used to obtain the magnetic sublevel $|j, m\rangle$ distribution of the final $\left(4 s 6 f^{1} F_{3}\right)$ state with respect to the dump laser polarization vector. This would result in a three-vector correlation experiment, where the three vectors are the initial relative velocity vector, the initial state polarization, and the dump laser polarization. This is analogous to several previous experiments, where the final state alignment is investigated with a probe laser exciting to a higher energy final state ${ }^{14,15}$ instead of dumping to a lower level.

The direct fluorescence method, however, does not reveal alignment information of the final state if the light collection is over a large angle and if no polarization selective optics are used. Basically, it constitutes a two-vector correlation where only the initial relative velocity vector and the 


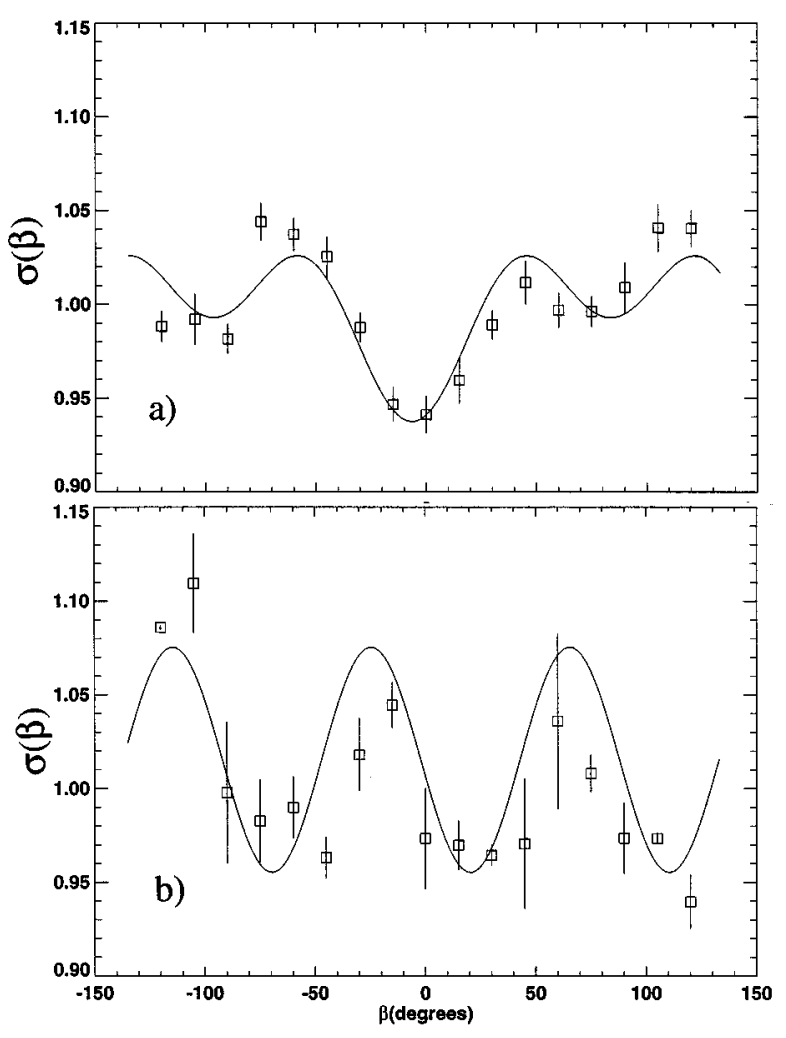

FIG. 6. Alignment curves for the energy transfer process $\mathrm{Ca}\left(4 s 7 d^{1} D_{2}\right)+\mathrm{He} \rightarrow \mathrm{Ca}\left(4 s 6 f^{1} F_{3}\right)+\mathrm{He}$ obtained by stimulated emission detection with laser polarizations (a) parallel and (b) perpendicular.

initial state polarization are manipulated relative to each other. In order to compare the dump method with the direct fluorescence, we choose a dumping scheme that allows all the final state magnetic sublevels to be probed equally. The final state $\left(4 s 6 f^{1} F_{3}\right)$ is dumped down to a $\left(4 s 4 d{ }^{1} D_{2}\right)$ state using an $1126 \mathrm{~nm}$ photon. The dump laser is elliptically polarized, which can be considered as a superposition of two circular polarizations, $\hat{e}_{-1}^{1}$ and $\hat{e}_{1}^{1}$. This means that for all magnetic sublevels, $|j=3, m\rangle$, one or two stimulated transitions to a lower magnetic sublevel, $|j=2, m \pm 1\rangle$, are possible. All $4 s 6 f$ magnetic sublevels are stimulated down to the $4 s 4 d$ levels only in the saturated limit. Thus our dump experiment is reduced to a two-vector correlation.

\section{B. Results}

\section{Stimulated emission detection}

The emission from the dumped state is collected and measured as a function of angle $\beta_{\text {rel }}$ and is shown in Figs. 6(a) and 6(b) for both parallel and perpendicular laser polarizations. Data were collected every $15^{\circ}$ to resolve fourfold structure. The error bars represent the average standard deviation of the mean in the points. In order to extract dynamical information about the energy transfer process, the following expression is used:

$$
\sigma(\beta)=\sum_{m}\left|\sum_{m^{\prime}} g_{m^{\prime}} d_{m m^{\prime}}^{j=2}\left(\beta_{\text {rel }}\right)\right|^{2} \sigma^{|m|},
$$

where $m$ and $m^{\prime}$ are the magnetic quantum numbers of the initial state with respect to the initial relative velocity vector and the preparation laser polarization, respectively. The Wigner $d_{m m^{\prime}}^{j}$ functions are rotation matrices which relate the quantization axes of the preparation frame and collision frame, where $v_{\text {rel }}$ serves as the quantization axis. Finally, the $g_{m}$ 'represent the population distribution of the initial state in the preparation frame. The angular behavior of the cross section may be written equivalently as

$$
\sigma\left(\beta_{\text {rel }}\right)=\sum_{n=0}^{j} a_{2 n} \cos \left(2 n \beta_{\text {rel }}\right) .
$$

The $\sigma^{|m|}$ are the cross sections for the transfer process of Eq. (17) for an initial $|j, m\rangle$ substate quantized with respect to relative velocity vector. These individual $\sigma^{|m|}$ cross sections are averages over the total impact parameter range, and averages over all magnetic sublevels of the final ${ }^{1} F_{3}$ state. The explicit expressions of Eq. (19) for the parallel and perpendicular laser polarization are provided in the literature. ${ }^{11}$ The solid curves in the alignment data represent a nonlinear least squares fit to Eqs. (18) and (19). In this experiment the initial state has the quantum number $j=2$, so up to fourfold structure may be observed. The data exhibit $\cos (2 \beta)$ and $\cos (4 \beta)$ structure. These contributions are related to the individual $m$ sublevels of the ${ }^{1} D_{2}$ initial state and relative values for the cross sections $\sigma^{|m|}$ are extracted from the alignment curves. These extracted values are normalized to $\sigma^{0}+2 \sigma^{|1|}+2 \sigma^{|2|}=5$. No new information is obtained from the perpendicular data and serves as a check on the relative cross sections obtained from the parallel data. The perpendicular data yield only $\sigma^{|1|}$ and the linear combination $3 \sigma^{0}+\sigma^{|2|}$, so only $\sigma^{|1|}$ values are listed along with the cross sections from the parallel data in Table I.

\section{Fluorescence detection}

Direct fluorescence intensity from the collisionally produced ${ }^{1} F_{3}$ state is measured at $411 \mathrm{~nm}$ as a function of the

TABLE I. Comparison of relative cross sections.

\begin{tabular}{ccccc}
\hline \hline \multirow{2}{*}{$\begin{array}{c}\text { Detection } \\
\text { method }\end{array}$} & Initial state & \multicolumn{3}{c}{ Relative $m$-sublevel cross sections ${ }^{\mathrm{a}}$} \\
\cline { 3 - 5 } & polarization & $\sigma^{0}$ & $\sigma^{|1|}$ & $\sigma^{|2|}$ \\
\hline Fluorescence & Parallel $(\uparrow \uparrow)$ & $0.952 \pm 0.005$ & $1.019 \pm 0.006$ & $1.021 \pm 0.006$ \\
Stimulated emission & Parallel $(\uparrow \uparrow)$ & $0.91 \pm 0.02$ & $1.05 \pm 0.02$ & $1.02 \pm 0.02$ \\
Fluorescence & Perpendicular $(\uparrow \leftrightarrow)$ & & $1.027 \pm 0.009$ & \\
Stimulated emission & Perpendicular $(\uparrow \leftrightarrow)$ & & $1.10 \pm 0.03$ & \\
\hline \hline
\end{tabular}

${ }^{a}$ Values normalized to $2 J+1=5$ for $J=2$. 


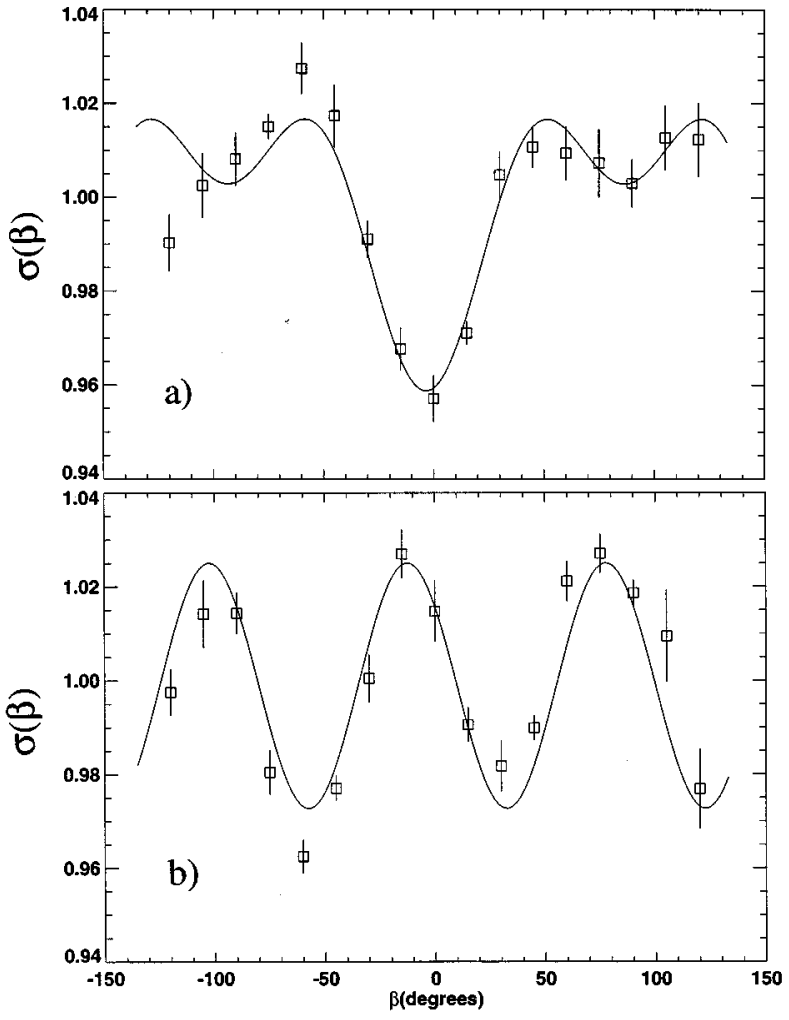

FIG. 7. Alignment curves for the energy transfer process $\mathrm{Ca}\left(4 s 7 d^{1} D_{2}\right)+\mathrm{He} \rightarrow \mathrm{Ca}\left(4 s 6 f^{1} F_{3}\right)+\mathrm{He}$ obtained by fluorescence detection with laser polarizations (a) parallel and (b) perpendicular.

angle $\beta_{\text {rel }}$. Figures $7(a)$ and $7(b)$ display the alignment curves for the two initial state laser preparations, parallel and perpendicular. The curves are fit to the expressions given above and the relative cross sections are given in Table I. The data for direct fluorescence detection is much less noisy than for the dump method, which we attribute to the fact that more signal is acquired with direct fluorescence than with the dump method. This may be because some of the population of the final state fluoresces before we pulse the dump laser, which decreases the amount of Rydberg levels stimulated down to the $4 s 4 d$ state.

\section{Discussion}

\section{Comparison of stimulated emission and fluorescence detection}

Table I provides a comparison of the $m$-sublevel cross sections obtained from the alignment curves for the two detection methods. The direct fluorescence data have smaller error bars because the signal to noise ratio was better than for the stimulated emission method. The comparison is quite good, with most values agreeing within the error bars of the stimulated emission data. We conclude that a two-vector correlation can be performed with the stimulated emission dump method provided the appropriate probing scheme is selected.

\section{Interpretation of relative $\boldsymbol{m}$-sublevel cross sections}

The dynamical information of the state-changing collision is contained in the individual $m$-sublevel cross sections.
These cross sections describe how the initial state symmetries (with respect to $v_{\text {rel }}$ ) contribute to the total cross section. From Table I, the $\sigma^{|2|}$ and $\sigma^{|1|}$ values are equal within experimental error and about $7 \%$ larger than the value of $\sigma^{0}$ (fluorescence data). The data can be analyzed with BornOppenheimer potential energy curves for the transient $\mathrm{CaHe}$ diatomic where the $\mathrm{Ca}{ }^{1} D_{2} m_{L}$ states $(0, \pm 1$, and \pm 2$)$ correlate asymptotically to the molecular $\Sigma, \Pi$, and $\Delta$ states. Unfortunately, there is no data on the qualitative or quantitative features of these potentials. However, even though the density of interacting states around the $4 s 7 d$ level is large, state specific effects are still observed. From the measured cross sections we conclude that the asymptotic $\Delta$ and $\Pi$ states are preferred in the collision event. The final state is a ${ }^{1} P_{1}$ and the asymptotic final states are $\Sigma$ and $\Pi$. This may mean that radially induced coupling between the initial and final $\Sigma$ states is not preferred. It is likely that angular (rotational) coupling of the initial $\Delta$ state to the final $\Pi$ state, radial coupling of the initial and final $\Pi$ states, and rotational coupling between the initial $\Pi$ and final $\Sigma$ states are important in this collisional transition. Additional theoretical work is needed to elucidate the state-changing mechanism for this system. In a previously studied ${ }^{1} D_{2}$ to ${ }^{1} F_{3}$ state change, ${ }^{11}$ it was found that $\sigma^{|2|} \gg \sigma^{0}>\sigma^{|1|}$. Compared to the preference observed here, most certainly the interaction potentials and the mechanism are quite different for the two ${ }^{1} D_{2} \rightarrow{ }^{1} F_{3}$ collisional transitions.

\section{SUMMARY}

We have shown that alignment effects observed by stimulated emission and direct fluorescence of an aligned state are essentially equivalent provided all the final state magnetic sublevels are probed equally. Therefore, this method can be applied to the study of two-vector correlations in collision-induced energy transfer of aligned Rydberg states. In the following paper we provide results for such an investigation. In addition, new results are obtained for a state-changing collision. Relative $m$-sublevel dependent cross sections for the $\left(4 s 7 d^{1} D_{2}\right)$ to $\left(4 s 6 f{ }^{1} F_{3}\right)$ energy transfer process are measured and qualitatively interpreted.

\section{ACKNOWLEDGMENTS}

The authors are grateful to the National Science Foundation for support of this research. We also acknowledge support from the NATO collaboration, Grant No. CRG 930833. The research of J.D. has been made possible by a fellowship of the Royal Netherlands Academy of Arts and Sciences.

${ }^{1}$ Rydberg States of Atoms and Molecules, edited by R. F. Stebbings and F. B. Dunning (Cambridge University Press, Cambridge, 1983), and references therein.

${ }^{2}$ T. F. Gallagher, S. A. Edelstein, and R. M. Hill, Phys. Rev. A 15, 1945 (1977).

${ }^{3}$ T. F. Gallagher, W. E. Cooke, and S. A. Edelstein, Phys. Rev. A 17, 904 (1978).

${ }^{4}$ T. F. Gallagher and W. E. Cooke, Phys. Rev. A 19, 2161 (1979).

${ }^{5}$ F. Gounand, P. R. Fournier, and J. Berlande, Phys. Rev. A 15, 2212 (1977).

${ }^{6}$ See, for example, F. G. Kellert, K. A. Smith, R. D. Rundel, F. B. Dunning, and R. F. Stebbings, J. Chem. Phys. 72, 317 (1980). 
${ }^{7}$ D. S. Thomson, M. J. Renn, and T. F. Gallagher, Phys. Rev. A 45, 358 (1992).

${ }^{8}$ M. J. Renn, W. R. Anderson, and T. F. Gallagher, Phys. Rev. A 49, 908 (1994).

${ }^{9}$ B. C. Saha and N. F. Lane, Phys. Rev. Lett. 72, 3487 (1994).

${ }^{10}$ W. Bussert, D. Neuschafer, and S. R. Leone, J. Chem. Phys. 87, 3833 (1987).

${ }^{11}$ R. L. Robinson, L. J. Kovalenko, C. J. Smith, and S. R. Leone, J. Chem. Phys. 92, 5360 (1990).

${ }^{12}$ J. P. J. Driessen, C. J. Smith, and S. R. Leone, J. Phys. Chem. 95, 8163 (1991).

${ }^{13}$ A. G. Suits, P. de Pujo, O. Sublemontier, J. P. Bisticot, J. Berlande, J. Cuvellier, T. Gustavsson, J. M. Mestdagh, P. Meynadier, and Y. T. Lee, J. Chem. Phys. 97, 4094 (1992).

${ }^{14}$ C. J. Smith, J. P. J. Driessen, L. Eno, and S. R. Leone, J. Chem. Phys. 96, 8212 (1992)

${ }^{15}$ C. J. Smith, E. M. Spain, M. J. Dalberth, J. P. J. Driessen, and S. R. Leone, J. Chem. Soc. Faraday Trans. 89, 1401 (1993).

${ }^{16}$ J. M. Mestdagh, J. P. Visticot, P. Meynadier, O. Sublemontier, and A. G. Suits, J. Chem. Soc. Faraday Trans. 89, 1413 (1993).

${ }^{17}$ T. L. D. Collins, A. J. McCaffery, and M. J. Wynn, Faraday Discuss. Chem. Soc. 91, 91 (1991)

${ }^{18}$ T. Duhoo and B. Pouilly, J. Chem. Phys. 101, 7554 (1994).

${ }^{19}$ J. P. J. Driessen and L. Eno, J. Chem. Phys. 97, 5532 (1992).

${ }^{20}$ J. P. J. Driessen and S. R. Leone, J. Phys. Chem. 96, 6136 (1992).
${ }^{21}$ L. J. Kovalenko, in The Chemical Dynamics and Kinetics of Small Radicals, edited by K. Liu and A. Wagner (to be published).

${ }^{22}$ L. J. Kovalenko, S. R. Leone, and J. B. Delos, J. Chem. Phys. 91, 6948 (1989).

${ }^{23}$ G. C. Schatz, L. J. Kovalenko, and S. R. Leone, J. Chem. Phys. 91, 6961 (1989).

${ }^{24}$ R. de Vivie-Riedle, J. P. J. Driessen, and S. R. Leone, J. Chem. Phys. 98, 2038 (1993).

${ }^{25}$ A. P. Hickman, S. Krebs, and W. E. Meyer, Phys. Rev. Lett. 72, 1814 (1994).

${ }^{26}$ B. J. Verhaar S. S. Op de Beek, H. C. W. Beijerinck, and J. P. J. Driessen, Phys. Rev. A (to be published).

${ }^{27}$ E. M. Spain, M. J. Dalberth, P. D. Kleiber, S. R. Leone, S. S. Op de Beek, and J. P. J. Driessen, J. Chem. Phys. 102, 9532 (1995).

${ }^{28}$ I. I. Fabrikant, Phys. Rev. A 48, R3411 (1994).

${ }^{29}$ C. E. Burkhardt and J. J Leventhal, Phys. Rev. A 43, 110 (1991).

${ }^{30}$ R. E. Olson, Phys. Rev. A 15, 631 (1977).

${ }^{31}$ K. B. MacAdam and M. A. Morrison, Phys. Rev. A 48, 1345 (1993).

${ }^{32}$ A. P. Hickman, R. E. Olson, and J. Pascale, in Rydberg States of Atoms and Molecules, Ref. 1, p. 187.

${ }^{33}$ E. Layton and M. A. Morrison (unpublished); W. Isaacs and M. A. Morrison (unpublished).

${ }^{34}$ A. Messiah, Quantum Mechanics (North-Holland, Amsterdam, 1961).

${ }^{35}$ R. N. Zare, Angular Momentum (Wiley, New York, 1988).

${ }^{36}$ Laurie J. Kovalenko, Ph.D thesis, University of Colorado, 1989. 